\title{
DYNAMICAL MASSES OF YOUNG M DWARFS: MASSES AND ORBITAL PARAMETERS OF GJ 3305 AB, THE WIDE BINARY COMPANION TO THE IMAGED EXOPLANET HOST 51 ERI*
}

\author{
Benjamin T. Montet ${ }^{1,2}$, Brendan P. Bowler ${ }^{1,9}$, Evgenya L. ShkolniK ${ }^{3,4}$, Katherine M. Deck ${ }^{1,9}$, Ji Wang ${ }^{1,5}$, \\ Elliott P. Horch ${ }^{6}$, Michael C. Liu ${ }^{7}$, Lynne A. Hillenbrand ${ }^{1}$, Adam L. Kraus ${ }^{8}$, and David Charbonneau ${ }^{2}$ \\ ${ }^{1}$ Cahill Center for Astronomy and Astrophysics, California Institute of Technology, Pasadena, CA 91125, USA; btm@astro.caltech.edu \\ ${ }^{2}$ Harvard-Smithsonian Center for Astrophysics, Cambridge, MA 02138, USA \\ ${ }^{3}$ School of Earth and Space Exploration, Arizona State University, Tempe, AZ 85287, USA \\ ${ }_{5}^{4}$ Lowell Observatory, 1400 West Mars Hill Road, Flagstaff, AZ 86001, USA \\ ${ }^{5}$ Department of Astronomy, Yale University, New Haven, CT 06511, USA \\ ${ }^{6}$ Department of Physics, Southern Connecticut State University, 501 Crescent Street, New Haven, CT 06515, USA \\ ${ }^{7}$ Institute for Astronomy, University of Hawaii, 2680 Woodlawn Drive, Honolulu, HI 96822, USA \\ ${ }^{8}$ Department of Astronomy, The University of Texas at Austin, Austin, TX 78712, USA \\ Received 2015 August 25; accepted 2015 October 8; published 2015 October 26
}

\begin{abstract}
We combine new high resolution imaging and spectroscopy from Keck/NIRC2, Discovery Channel Telescope/ DSSI, and Keck/HIRES with published astrometry and radial velocities to measure individual masses and orbital elements of the GJ 3305 AB system, a young ( 20 Myr) M+M binary (unresolved spectral type M0) member of the $\beta$ Pictoris moving group comoving with the imaged exoplanet host 51 Eri. We measure a total system mass of $1.11 \pm 0.04 M_{\odot}$, a period of $29.03 \pm 0.50$ year, a semimajor axis of $9.78 \pm 0.14 \mathrm{AU}$, and an eccentricity of $0.19 \pm$ 0.02. The primary component has a dynamical mass of $0.67 \pm 0.05 M_{\odot}$ and the secondary has a mass of $0.44 \pm$ $0.05 M_{\odot}$. The recently updated BHAC15 models are consistent with the masses of both stars to within $1.5 \sigma$. Given the observed masses the models predict an age of the GJ 3305 AB system of $37 \pm 9$ Myr. Based on the observed system architecture and our dynamical mass measurement, it is unlikely that the orbit of 51 Erib has been significantly altered by the Kozai-Lidov mechanism.
\end{abstract}

Key words: astrometry - binaries: close - stars: fundamental parameters - stars: individual (GJ 3305 AB)

\section{INTRODUCTION}

Loose associations of young, nearby $(<70 \mathrm{pc})$ stars with common ages, kinematics, and origins have been a subject of increasing interest (Zuckerman et al. 2004; Shkolnik et al. 2012; Malo et al. 2013). Because of their proximity to Earth, these young moving groups (YMGs) are excellent targets to study premain-sequence (PMS) stellar and substellar evolution, protoplanetary and debris disk structure, and giant planet formation at ages between distant star-forming regions and old field stars (e.g., Close et al. 2005; Nielsen \& Close 2010). About 10 YMGs containing hundreds of objects between 8 and 120 million years old are known (e.g., Torres et al. 2008).

As these moving groups are amenable to numerous age dating methods, including kinematic techniques, they provide the opportunity to measure dynamical masses of PMS lowmass binary objects and test stellar evolution models (Stassun et al. 2014). Generally, PMS stellar masses are inferred by comparing a star's temperature, luminosity and metallicity to model predictions (e.g., Schaefer et al. 2014). These models are poorly constrained by observations and may induce systematic offsets (Dupuy et al. 2009, 2014). Worse yet, different models predict disparate masses, primarily due to uncertainties in the treatment of convection in low-gravity atmospheres (Baraffe et al. 2002), stellar accretion history (Baraffe \& Chabrier 2010), and molecular line lists (Baraffe et al. 2015). In some cases, model-predicted masses can differ by a factor of two or more

\footnotetext{
* Some of the data presented herein were obtained at the W. M. Keck Observatory, which is operated as a scientific partnership among the California Institute of Technology, the University of California and the National Aeronautics and Space Administration. The Observatory was made possible by the generous financial support of the W. M. Keck Foundation.

${ }^{9}$ Caltech Joint Center for Planetary Astronomy Fellow.
}

(Hillenbrand \& White 2004; Schlieder et al. 2014). Dynamical mass measurements of binary stars with known ages are essential to test models.

Recently, Macintosh et al. (2015) presented 51 Eri b, the first exoplanet discovery from the Gemini Planet Imager. The planet has a mass of $\approx 2 M_{\text {Jup }}$ (assuming a hot start model), a projected separation of $13 \mathrm{AU}$, a temperature of 600-750 K, and a T4.5T6 spectral type. GJ 3305 is known to be a binary with combined spectral type M0 (Kasper et al. 2007). Feigelson et al. (2006) identified GJ 3305 and 51 Eri as an F0-M0 common proper motion pair, separated by $66^{\prime \prime}$ or $\sim 2000 \mathrm{AU}$.

As a binary system, a dynamical mass can be measured for both stars in GJ $3305 \mathrm{AB}$. As both stars are members of the $\beta$ Pictoris moving group, an approximate age of the system is known (24 \pm 3 Myr; Binks \& Jeffries 2014; Mamajek \& Bell 2014; Bell et al. 2015). While most dynamical masses of $\mathrm{M}$ dwarfs are limited by distance uncertainties, 51 Eri has a parallax from Hipparcos measured to a precision of $1 \%$. Combining this parallax with 15 years of imaging and RV data enables us to determine the system orbital parameters, elucidating the architecture of this 4-or more-body system.

In this paper, we combine RV and astrometric observations of GJ $3305 \mathrm{AB}$ to measure orbital parameters and masses for each star. We compare these masses to model predictions and discuss the possible implications of this binary pair on the longterm evolution of the orbit of 51 Eri b.

\section{DATA COLLECTION AND REDUCTION}

GJ $3305 \mathrm{AB}$ has been imaged and resolved many times (Kasper et al. 2007; Bergfors et al. 2010; Delorme et al. 2012; Janson et al. 2012, 2014). The system was also imaged with NIRC2 (Wizinowich et al. 2000) in one unpublished epoch in 2001 
Table 1

Data for GJ $3305 \mathrm{AB}$

\begin{tabular}{|c|c|c|c|c|c|c|}
\hline $\begin{array}{l}\text { Epoch } \\
\text { (Year) }\end{array}$ & Bandpass & $\begin{array}{c}\mathrm{RV} \\
\left(\mathrm{km} \mathrm{s}^{-1}\right)\end{array}$ & $\begin{array}{l}\text { Contrast } \\
(\Delta \mathrm{mag})\end{array}$ & $\begin{array}{l}\text { Separation } \\
\text { (mas) }\end{array}$ & $\begin{array}{l}\text { Position Angle } \\
\text { (deg) }\end{array}$ & Source \\
\hline 2001.910 & $\mathrm{H}_{2}(\nu=1-0)$ & $\ldots$ & $1.00 \pm 0.02$ & $286 \pm 1$ & $198.1 \pm 0.1$ & This Work \\
\hline 2002.162 & $H$ & $\cdots$ & $1.02 \pm 0.02$ & $275.4 \pm 1.5$ & $197.9 \pm 0.2$ & This Work \\
\hline 2003.195 & $H$ & $\cdots$ & $0.99 \pm 0.01$ & $217 \pm 1$ & $196.8 \pm 0.1$ & This Work \\
\hline 2004.02 & $L^{\prime}$ & $\cdots$ & $\cdots$ & $159 \pm 2$ & $194 \pm 1$ & Delorme et al. (2012) \\
\hline 2004.95 & $L^{\prime}$ & $\cdots$ & $0.88 \pm 0.28$ & $93 \pm 2$ & $189.5 \pm 0.4$ & Kasper et al. (2007) \\
\hline 2009.13 & SDSS $i^{\prime}+z^{\prime}$ & $\cdots$ & $\cdots$ & $231 \pm 2$ & $19.2 \pm 0.3$ & Janson et al. (2012) \\
\hline 2009.90 & $L^{\prime}$ & $\cdots$ & $\cdots$ & $269 \pm 3$ & $18.6 \pm 1.0$ & Delorme et al. (2012) \\
\hline 2009.98 & $L^{\prime}$ & $\cdots$ & $\cdots$ & $272 \pm 3$ & $19.2 \pm 1.0$ & Delorme et al. (2012) \\
\hline 2010.10 & SDSS $z^{\prime}$ & $\cdots$ & $1.34 \pm 0.01$ & $284 \pm 3$ & $18.5 \pm 0.6$ & Janson et al. (2012) \\
\hline 2010.10 & $\operatorname{SDSS} i^{\prime}$ & $\cdots$ & $3.73 \pm 0.01$ & $\ldots$ & $\ldots$ & Janson et al. (2012) \\
\hline 2010.81 & SDSS $z^{\prime}$ & $\ldots$ & $\ldots$ & $297 \pm 3$ & $19.4 \pm 0.3$ & Janson et al. (2014) \\
\hline 2014.746 & DSSI $R$ & $\cdots$ & $1.89 \pm 0.04$ & $239 \pm 1$ & $16.4 \pm 0.2$ & This Work \\
\hline 2014.746 & DSSI $I$ & $\cdots$ & $1.17 \pm 0.03$ & $240 \pm 1$ & $16.1 \pm 0.2$ & This Work \\
\hline 2015.653 & $K$ & $\cdots$ & $0.93 \pm 0.01$ & $199 \pm 1$ & $15.6 \pm 0.1$ & This Work \\
\hline 2015.653 & $H$ & $\cdots$ & $0.99 \pm 0.01$ & $198 \pm 1$ & $15.6 \pm 0.1$ & This Work \\
\hline 2015.653 & $J$ & $\cdots$ & $0.97 \pm 0.01$ & $199 \pm 1$ & $15.6 \pm 0.2$ & This Work \\
\hline 2015.653 & $Y$ & $\cdots$ & $1.06 \pm 0.03$ & $200 \pm 1$ & $15.6 \pm 0.1$ & This Work \\
\hline 2003.796 & HIRES $V$ & $19.41 \pm 0.38$ & $\cdots$ & $\cdots$ & $\cdots$ & This work \\
\hline 2004.884 & NIRSPEC $K$ & $19.86 \pm 0.05$ & $\ldots$ & $\ldots$ & $\cdots$ & Bailey et al. (2012) \\
\hline 2005.862 & NIRSPEC $K$ & $20.55 \pm 0.06$ & $\cdots$ & $\cdots$ & $\cdots$ & Bailey et al. (2012) \\
\hline 2005.971 & HIRES $V$ & $21.70 \pm 0.30$ & $\cdots$ & $\cdots$ & $\cdots$ & Shkolnik et al. (2012) \\
\hline 2006.014 & NIRSPEC $K$ & $20.82 \pm 0.05$ & $\cdots$ & $\cdots$ & $\ldots$ & Bailey et al. (2012) \\
\hline 2006.016 & NIRSPEC $K$ & $20.95 \pm 0.05$ & $\cdots$ & $\cdots$ & $\cdots$ & Bailey et al. (2012) \\
\hline
\end{tabular}

Notes. In some previous analyses, contrast ratios were not listed for specific epochs. Observations without listed separations correspond to simultaneous multiband photometry.

${ }^{a}$ Observations published without uncertainty estimates; we choose conservative values.

available in the Keck Observatory Archives (KOA; PI Zuckerman). In this work, we combine these data with five observations from 2002 to 2015, three using Keck/NIRC2 and one with the Differential Speckle Survey Instrument (DSSI; Horch et al. 2009) at the Discovery Channel Telescope at Lowell Observatory.

All NIRC2 data were obtained with the narrow camera mode, which has a field of view of 10 ". $2 \times 10$ ". 2 and a plate scale of 9.952 mas pixel $^{-1}$ (Yelda et al. 2010). All images were flat fielded and cleaned of bad pixels and cosmic rays. Astrometry and relative photometry of GJ 3305 was derived by simultaneously fitting three bivariate Gaussians to each component following Liu et al. (2010).

DSSI allows for simultaneous observations in two filters. We use the DSSI $R$ and $I$ filters, with central wavelengths 692 and $880 \mathrm{~nm}$ and FWHMs of 40 and $50 \mathrm{~nm}$. We obtained 1000 40ms exposures in each channel simultaneously. The data were then reduced following Horch et al. (2015). Specifically, the autocorrelation of each frame was calculated and summed over all exposures, and the near-axis subplanes of the image bispectrum were calculated. To create a reconstructed image, the Fourier transform of the autocorrelation of the binary was divided by that of a nearby point source (HR 1415). The square root of this value is taken, and the result combined with a phase function derived from the bispectral subplanes. The pixel scale (19 mas pixel ${ }^{-1}$ in $R$ and 20 mas pixel $^{-1}$ in $\left.I\right)$ and orientation of the detector were found by observing several widely separated binaries with known astrometry. Our astrometry is listed in Table 1.

The GJ 3305 binary system has also been monitored spectroscopically. One Keck/HIRES spectrum from 2003 exists in the KOA (PI Zuckerman); we measure the RV following Kraus et al. (2015). We combine this spectrum with nine additional spectra from Bailey et al. (2012), Shkolnik et al. (2012), and Elliott et al. (2014). In all cases, the RVs were calculated treating the system as an SB1. We take the reported $\mathrm{RV}$ and uncertainty for each observation, but assume the flux from the secondary is non-negligible, as explained in Section 3.

\section{ANALYSIS}

We infer the orbital parameters of GJ 3305 AB by comparing the astrometric and RV data to a Keplerian orbit model at each of the observation times. A parallax, astrometric orbit, and SB1 

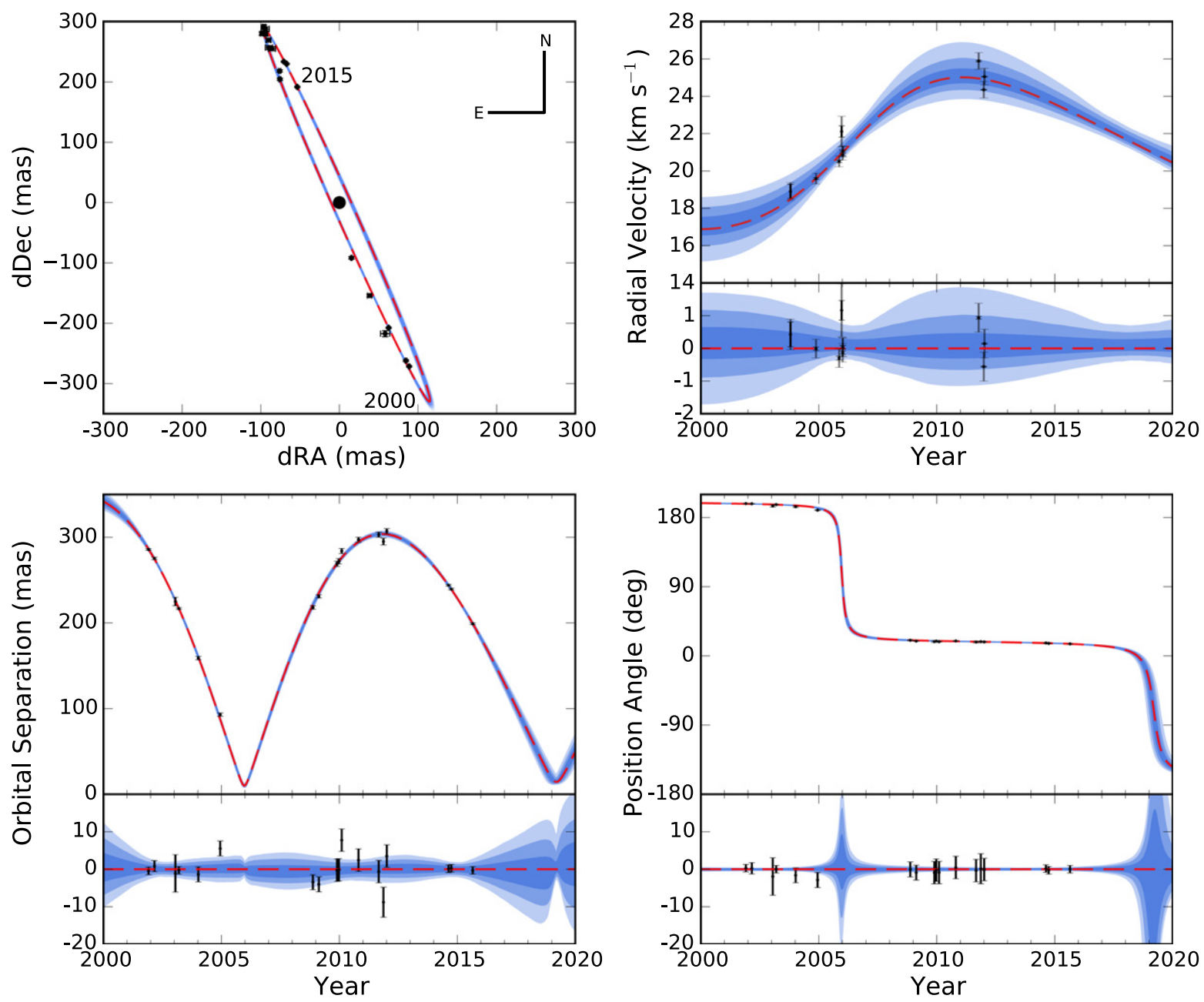

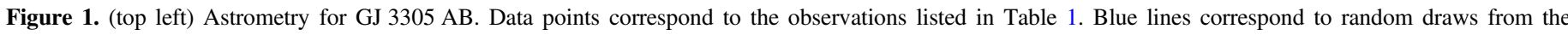

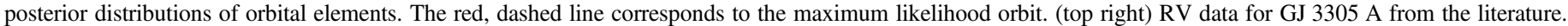



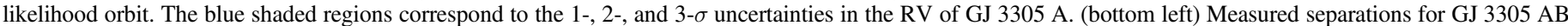

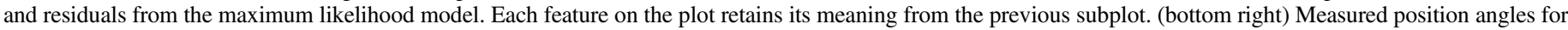
GJ $3305 \mathrm{AB}$ and residuals from the maximum likelihood model.

RV data can be combined to measure individual masses of each star (e.g., Bean et al. 2007). There is no measured parallax for GJ 3305, so we adopt the Hipparcos distance to 51 Eri/ HIP 21547: $29.43 \pm 0.30 \mathrm{pc}$ (van Leeuwen 2007). These two comoving systems have a projected separation of $1940 \pm$ $20 \mathrm{AU}$, or $0.01 \mathrm{pc}$. It is unlikely that the radial distance between the two could be significantly larger while remaining bound; we apply this parallax as a prior on the distance to GJ 3305 .

We then fit for nine additional parameters that define the orbits of the two stars as viewed from Earth. Of these, seven can be obtained from astrometry. These parameters are the eccentricity vectors $\sqrt{e} \cos \omega$ and $\sqrt{e} \sin \omega$, the time of periapse $t_{P}$, the period $P$, the total mass $M_{1}+M_{2}$, the inclination $i$, and the longitude of the ascending node $\Omega$. We parameterize the eccentricity vector in this manner following Eastman et al. (2013). The RV data can provide additional information about several of these (not $M_{1}+M_{2}$ or $\Omega$ directly), also allowing us to fit the systemic RV $\gamma$ and the secondary mass $M_{2}$.

We include ten additional terms to account for possible systematics in the datas. This star has been imaged, resolved, and published by four different groups. We account for the possibility each group may have underestimated their uncertainties on the orbital separation and position angle by a multiplicative factor by including a systematic error term on the measured positions from each group, allowing outlier points to be downweighted without manually choosing specific points to downweight. We do the same with our reductions of both archival and new data, allowing for separate systematic error terms on our data from Keck/NIRC2 and DCT/DSSI, providing a total of six systematic error terms. We allow the uncertainties on each dataset to be inflated up to a factor of five.

Similarly, we allow for the possibility that the uncertainties in the RVs may be underestimated, possibly due to stellar variability (Moulds et al. 2013), errors in systemic RVs of standard stars, or drifts in the stability of the spectrographs. As our RV data originate from four sources, we allow each to have its own systematic error term, analogous to the jitter term commonly applied in RV orbit fits of exoplanets (e.g., Johnson et al. 2011):

$$
\log \mathcal{L} \propto-\sum_{i}\left[\log \sqrt{\sigma_{o, i}^{2}+\sigma_{s}^{2}}+0.5\left(\frac{\left(f_{i}(t)-v_{i}(t)\right)^{2}}{\sigma_{o, i}^{2}+\sigma_{s}^{2}}\right)\right] .
$$


Here, $\mathcal{L}$ is the likelihood of the data given some underlying physical model, $\sigma_{o, i}$ is the observed uncertainty on the $i$ th data point, $\sigma_{s}$ the systematic error associated with each particular set of observations, $f_{i}(t)$ the RV model evaluated at time $t$, and $v_{i}(t)$ the observed RV at each $t$. Maximum likelihood jitter values range from $0.13 \mathrm{~km} \mathrm{~s}^{-1}$ for the 2003 HIRES data to $0.57 \mathrm{~km} \mathrm{~s}^{-1}$ for the UVES data, suggesting stellar jitter is significant in the RV data, as expected for young stars.

In all cases, one set of lines are observed because the RV separation is smaller than the line width. We expect each RV measurement to be the flux-weighted sum of the two individual RVs. At each step, we calculate the RVs for each star, weighting them according to their expected flux contribution in each bandpass, using the observed flux ratios in the visible and near-IR as priors and assuming an additional $0.1 \mathrm{mag}$ of variability in the optical and $0.05 \mathrm{mag}$ in the near-IR.

We neglect the possibility that 51 Eri could contribute significantly to the observed RV signal. Following Equation (1) of Montet et al. (2015), the maximum RV acceleration expected from $51 \mathrm{Eri}$ is $3 \mathrm{~cm} \mathrm{~s}^{-1} \mathrm{yr}^{-1}$, well below our sensitivity.

We calculate posterior distributions for all parameters using emcee (Foreman-Mackey et al. 2013), an implementation of the affine-invariant Markov Chain Monte Carlo ensemble sampler of Goodman \& Weare (2010). After performing a local optimization to determine a maximum-likelihood fit, we move 3000 walkers each 4000 steps. We discard the first 2000 steps of each walker as burn-in, and use the test of Geweke (1992) and visual inspection to verify the system has converged. The data and allowed orbits are shown in Figure 1. Summary statistics for the orbital parameters are given in Table 2. We note the fitted systemic RV of $20.76 \pm 0.18 \mathrm{~km} \mathrm{~s}^{-1}$ is consistent with the measured RV for 51 Eri, $21.0 \pm$ $1.2 \mathrm{~km} \mathrm{~s}^{-1}$ (Bobylev 2006) and the UVW velocities are consistent with Mamajek \& Bell (2014). Our samples are available online. $^{10}$

We estimate bolometric luminosities for both stars by integrating the CFHIST2011_2015 model spectra of Baraffe et al. (2015). We use the 3700 and $3500 \mathrm{~K}$ models with $\log g=4.5$ (cgs) as spectral templates, scaling them until they match the observed combined and differential magnitudes in each available bandpass. We add in quadrature 0.10 mag of uncertainty in our visible-light magnitudes and 0.05 mag in the near-IR to account for stellar variability.

\section{COMPARISON WITH BHAC15 EVOLUTIONARY MODELS}

Given the known distance to the system from Hipparcos we can test if theoretical stellar evolution models accurately predict the inferred stellar masses and age of the $\beta$ Pic moving group. Combined-light photometry spanning from $B(0.4 \mu \mathrm{m})$ to $K s$ $(2.3 \mu \mathrm{m})$ was measured by the APASS, 2MASS, and WISE surveys (Table 3). We add an uncertainty of $0.03 \mathrm{mag}$ in quadrature to the listed APASS uncertainties due to the presence of systematics in APASS DR9 at that level (Henden et al. 2012). We also have obtained one epoch of differential photometry in two visible-light bandpasses with DSSI and two near-IR bandpasses $(H$ and $\mathrm{Br} \gamma)$ with Keck/NIRC2.

\footnotetext{
${ }^{10}$ http://www.astro.caltech.edu/ btm/research/gj3305.html
}

Table 2

Parameters for GJ 3305 AB

\begin{tabular}{lccc}
\hline \hline Parameter & Median & & $\begin{array}{c}\text { Uncertainty } \\
(1 \sigma)\end{array}$ \\
\hline$\sqrt{e} \cos \omega$ & 0.160 & \pm & 0.019 \\
$\sqrt{e} \sin \omega$ & -0.406 & \pm & 0.015 \\
Eccentricity & 0.19 & \pm & 0.02 \\
Argument of Periastron $\omega(\mathrm{deg})$ & -69 & \pm & 3 \\
Time of Periastron $($ Year $)$ & 2007.14 & \pm & 0.16 \\
Orbital Period (Year) & 29.03 & \pm & 0.50 \\
GJ 3305 A Mass $\left(M_{\odot}\right)$ & 0.67 & \pm & 0.05 \\
GJ 3305 B Mass $\left(M_{\odot}\right)$ & 0.44 & \pm & 0.05 \\
Total System Mass $\left(M_{\odot}\right)$ & 1.11 & \pm & 0.04 \\
Mass Ratio $M_{B} / M_{A}$ & 0.65 & \pm & 0.10 \\
Orbital Inclination, $i(\mathrm{deg})$ & 92.1 & \pm & 0.2 \\
Orbital Semimajor Axis, $a(\mathrm{AU})$ & 9.78 & \pm & 0.14 \\
Long. of Ascending Node, $\Omega(\mathrm{deg})$ & 18.8 & \pm & 0.2 \\
Systemic RV Velocity, $\gamma\left(\mathrm{km} \mathrm{s}{ }^{-1}\right)$ & 20.76 & \pm & 0.18 \\
RV semiamplitude $K_{A}\left(\mathrm{~km} \mathrm{~s}{ }^{-1}\right)$ & 4.01 & \pm & 0.38 \\
U (km s ${ }^{-1}$ ) & -13.76 & \pm & 0.24 \\
V (km s ${ }^{-1}$ ) & -16.40 & \pm & 0.40 \\
W (km s ${ }^{-1}$ ) & -9.71 & \pm & 0.36 \\
GJ 3305 A Luminosity $\left(L_{\odot}\right)$ & 0.112 & \pm & 0.007 \\
GJ 3305 B Luminosity $\left(L_{\odot}\right)$ & 0.043 & \pm & 0.005 \\
\hline
\end{tabular}

Table 3

Photometry for GJ 3305 AB

\begin{tabular}{lccc}
\hline \hline Bandpass & Source & Magnitude & Uncertainty \\
\hline Combined & & & \\
$B$ & APASS DR9 & 11.94 & 0.03 \\
$V$ & APASS DR9 & 10.56 & 0.05 \\
$g^{\prime}$ & APASS DR9 & 11.27 & 0.03 \\
$r^{\prime}$ & APASS DR9 & 10.03 & 0.07 \\
$J$ & 2MASS & 7.30 & 0.02 \\
$H$ & 2MASS & 6.64 & 0.05 \\
$K$ & 2MASS & 6.41 & 0.02 \\
$W 1$ & $W I S E$ & 6.34 & 0.03 \\
$W 2$ & $W I S E$ & 6.21 & 0.02 \\
$W 3$ & $W I S E$ & 6.16 & 0.02 \\
$W 4$ & $W I S E$ & 6.00 & 0.04 \\
\hline $\mathrm{Resolved}$ & & & \\
$\Delta 692$ & DSSI & 1.89 & 0.04 \\
$\Delta 880$ & DSSI & 1.17 & 0.03 \\
$\Delta \mathrm{H}_{2}$ & Keck/NIRC2 & 1.00 & 0.02 \\
$\Delta \mathrm{Br} \gamma$ & Keck/NIRC2 & 0.92 & 0.01 \\
$\Delta H$ & Keck/NIRC2 & 1.00 & 0.02 \\
\hline
\end{tabular}

We compare the observed brightness of GJ $3305 \mathrm{AB}$ to that predicted by the BHAC15 models of Baraffe et al. (2015) for two stars of masses consistent with those inferred during our analysis as a function of age. We find models of $25 \mathrm{Myr}$ old stars accurately predict the combined-light near-IR flux for these stars, although the models predict brighter $V$ magnitudes than those observed (Figure 2). However, star $B$ is brighter than these same models predict: a 25 Myr old GJ 3305 B would be significantly brighter than what is observed. Assuming the stars are coeval, the models then predict a mass for GJ $3305 \mathrm{~B}$ that is $20 \%$ lower than the observed mass.

We create a simulated spectral energy distribution for each star, given the measured masses and the average age of $\beta$ Pic as measured from higher-mass stars. We interpolate absolute 

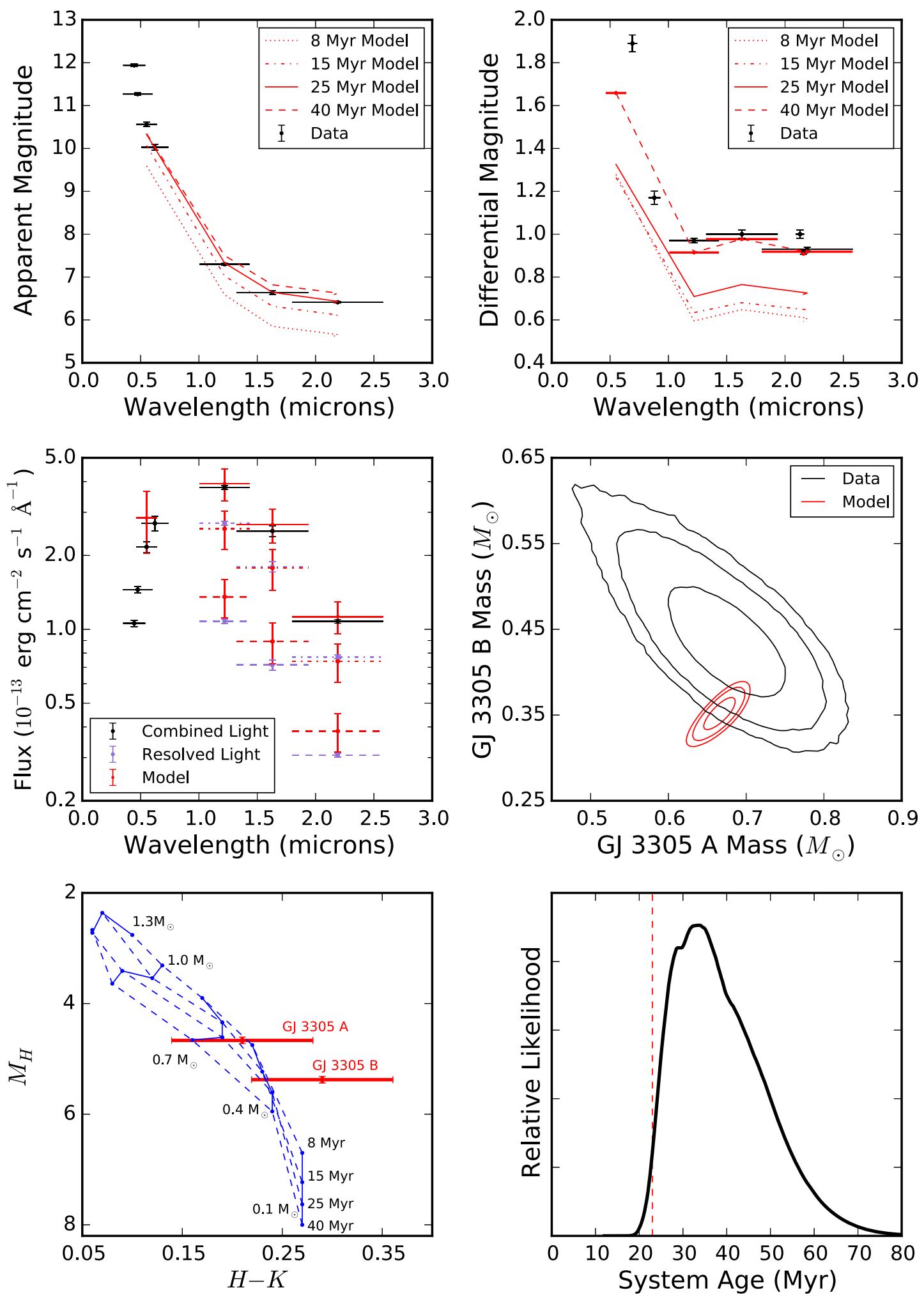

Figure 2. Top: (Left) Combined-light, unresolved and (Right) differential, resolved photometry for GJ 3305 AB (black) compared to predictions (red) of the BHAC15 models as a function of age given the observed masses and parallax. The data are consistent with an age larger than 25 Myr. Plotted bars along the abscissa correspond to the width of each filter and are meant to guide the eye: they do not represent an uncertainty. (Middle left) SED for the system, assuming a $24 \pm 3 \mathrm{Myr}$ age and the observed masses. Combined-light photometry is in black and resolved photometry in purple. While the model accurately reproduces the observed flux from GJ 3305 A, it overpredicts the received flux from GJ 3305 B. (Middle right) Joint posterior probability distributions on the masses of the two stars, (black) inferred from the astrometry and RV data and (red) predicted by the BHAC15 models given the observed combined-light and differential photometry assuming an age of $24 \pm$ 3 Myr. Contours correspond to the 1,2, and $3 \sigma$ confidence regions. The BHAC15 models predict a mass for GJ $3305 \mathrm{~B}$ consistent with the mass inferred from the data, but underpredicts the mass of GJ 3305 A by 20\%. (Bottom left) CMD showing the absolute $H$ magnitudes and $H-K$ colors of GJ 3305 AB compared to theoretical models. The models provide a more accurate fit for GJ 3305 A than GJ 3305 B. (Bottom right) Posterior probability distribution on the age of the GJ 3305 system, calculated by marginalizing the joint mass-age posterior over all allowed masses, assuming both stars are the same age. The BHAC15 models predict an age of $37 \pm 9$ Myr; the dashed line represents the Bell et al. (2015) age of the $\beta$ Pictoris system. 
magnitudes predicted by the updated BHAC 15 models of Baraffe et al. (2015) along isochrones and isomass contours to predict apparent magnitudes for these stars in each bandpass. We find that the total received flux is lower than predicted by the BHAC15 models in each bandpass. While the flux for GJ 3305 A is consistent with the model predictions, GJ 3305 B is fainter than predicted.

Given the observed masses, we then vary the age of the system, assuming both stars are coeval, to determine which system age would be predicted by these models given the observed combined and differential magnitudes. We apply a flat prior on the age of the system, finding the BHAC15 models predict an age of $37 \pm 9 \mathrm{Myr}$, consistent with the overall age of the moving group ( $24 \pm 3 \mathrm{Myr}$, Bell et al. 2015). As the system is unambiguously young, we can also confirm 51 Erib as a planetary mass object.

\section{DISCUSSION}

We have measured the masses and orbits of GJ $3305 \mathrm{AB}$, finding both to be consistent with the BHAC15 models at the $1.5 \sigma$ level. In the future GJ $3305 \mathrm{AB}$ and the gravitationally bound $51 \mathrm{Eri} \mathrm{Ab}$ will be able to act as an isochronal test as a coeval, co-metallicity quadruple system spanning stellar to planetary mass regimes.

The derived period of GJ 3305 (29.03 \pm 0.50 year) is longer than the 21 year found by Delorme et al. (2012). The authors of that paper did not have sufficient data to fit all orbital parameters, so they fixed the total system mass to $1.3 M_{\odot}$. Given our lower mass measurement, it is not surprising that our measured orbital period is longer.

\subsection{Current Limitations}

It is possible that an unseen very low-mass star or brown dwarf orbiting GJ 3305 B could cause us to overestimate its mass, causing the observed $20 \%$ discrepancy. For the system to be stable over $20 \mathrm{Myr}$, such a companion would have to be in a close $(P<50$ day) orbit. The companion would then have to be in a nearly face-on $\left(i<10^{\circ}\right)$ orbit to evade RV detection. Such companions could be found through continued astrometric monitoring of GJ 3305. Such a companion would not affect our astrometry due to its small separation from GJ $3305 \mathrm{~B}$ and would likely not affect our photometry due to its low luminosity relative to the other stars in the system.

Most PMS M dwarfs have distance measurements to a precision no better than 5\%, meaning the total mass cannot be measured to better than $15 \%$ (e.g., Shkolnik et al. 2012). The uncertainty in the mass of $\mathrm{GJ} 3305 \mathrm{AB}$ is only $4 \%$ : the dominant source of uncertainty in this value is the $1 \%$ Hipparcos parallax to 51 Eri, making this system an ideal low-mass benchmark. With a Gaia parallax forthcoming in the next few years, parallaxes for low-mass PMS stars will be improved substantially. Long-term astrometric and RV monitoring of wide $\mathrm{M}$ dwarfs is essential as parallaxes are obtained over the next few years.

The uncertainty in the individual mass of each star is dominated by the uncertainty in the Doppler semiamplitude. While additional astrometric observations will not significantly improve the measured physical properties of GJ 3305, additional RV observations will be important. RV observations behind $\mathrm{AO}$ would be especially beneficial, as the RV from each star could be measured separately, instead of a flux-weighted RV centroid.

\subsection{Dynamical Effects on 51 Erib}

GJ $3305 \mathrm{AB}$ and 51 Eri $\mathrm{Ab}$ exist in a dynamical configuration that may be susceptible to Kozai-Lidov oscillations (Kozai 1962; Lidov 1962), as suggested by Macintosh et al. (2015). In this scenario, the planet-star binary (51 Eri Ab) interacts secularly with $\mathrm{GJ} 3305 \mathrm{AB}$, leading to oscillations in inclination and eccentricity of the planet-star sub-system. The timescale for such an interaction is

$$
\tau \approx P_{\text {planet }} \frac{M_{\star}}{M_{\text {pert }}}\left(\frac{a_{\text {pert }}}{a_{\text {planet }}}\right)^{3}\left(1-e_{\text {pert }}^{2}\right)^{3 / 2}
$$

where $P_{\text {planet }}$ is the orbital period of a planet with a semimajor axis of $a_{\text {planet }}$ about a host of mass $M_{\star}, M_{\text {pert }}$ is the mass of a distant perturber, and $a_{\text {pert }}$ and $e_{\text {pert }}$ are the semimajor axis and eccentricity of the perturber/planet-star "binary" orbit (see, e.g., Holman et al. 1997).

Although we have limited information about this system, we can estimate the timescale for Kozai-Lidov cycles should the mutual inclination of the 51 Eri Ab system and (51 Eri Ab)(GJ $3305 \mathrm{AB}$ ) system satisfy $140^{\circ} \lesssim i_{m} \gtrsim 40^{\circ}$. Taking the instantaneous sky-projected separations as a proxy for the semimajor axes and inferred masses of $M_{\star}=1.75 M_{\odot}$ (Simon \& Schaefer 2011) and $M_{\text {pert }}=1.1 M_{\odot}$ yields a timescale of $\tau \sim 2 \times 10^{8}$ year $\left(1-e_{\text {pert }}^{2}\right)^{3 / 2}$. Therefore, unless the eccentricity of GJ 3305 about the 51 Eri subsystem satisfies $e_{\text {pert }} \gtrsim$ 0.9 , the timescale for Kozai-Lidov oscillations is longer than the age of the system, so we do not expect the Kozai-Lidov mechanism to have had time to induce a large eccentricity or spin-orbit misalignment within the 51 Eri sub-system. If future observations indicate non-zero spin-orbit misalignment or a high eccentricity for the orbit of 51 Eri b, a primordial origin unrelated to the distant perturbers would be suggested.

B.T.M. is supported by the National Science Foundation Graduate Research Fellowship under grant No. DGE-1144469.

This research has made use of the Keck Observatory Archive (KOA), which is operated by the W. M. Keck Observatory and the NASA Exoplanet Science Institute (NExScI), under contract with the National Aeronautics and Space Administration.

These results made use of Lowell Observatory's Discovery Channel Telescope. Lowell operates the DCT in partnership with Boston University, Northern Arizona University, the University of Maryland, and the University of Toledo. Partial support of the DCT was provided by Discovery Communications.

This publication was made possible through the support of a grant from the John Templeton Foundation. The opinions expressed in this publication are those of the authors and do not necessarily reflect the views of the John Templeton Foundation.

The authors wish to recognize and acknowledge the very significant cultural role and reverence that the summit of Maunakea has always had within the indigenous Hawaiian community. We are most fortunate to have the opportunity to conduct observations from this mountain.

Facilities: DCT:DSSI, Keck:I (HIRES), Keck:II (NIRC2). 


\section{REFERENCES}

Bailey, J. I., III, White, R. J., Blake, C. H., et al. 2012, ApJ, 749, 16 Baraffe, I., \& Chabrier, G. 2010, A\&A, 521, A44

Baraffe, I., Chabrier, G., Allard, F., \& Hauschildt, P. H. 2002, A\&A, 382, 563

Baraffe, I., Homeier, D., Allard, F., \& Chabrier, G. 2015, A\&A, 577, A42

Bean, J. L., McArthur, B. E., Benedict, G. F., et al. 2007, AJ, 134, 749

Bell, C. P. M., Mamajek, E. E., \& Naylor, T. 2015, MNRAS, 454, 593

Bergfors, C., Brandner, W., Janson, M., et al. 2010, A\&A, 520, A54

Binks, A. S., \& Jeffries, R. D. 2014, MNRAS, 438, L11

Bobylev, V. V. 2006, AstL, 32, 816

Close, L. M., Lenzen, R., Guirado, J. C., et al. 2005, Natur, 433, 286

Delorme, P., Lagrange, A. M., Chauvin, G., et al. 2012, A\&A, 539, A72

Dupuy, T. J., Liu, M. C., \& Ireland, M. J. 2009, ApJ, 692, 729

Dupuy, T. J., Liu, M. C., \& Ireland, M. J. 2014, ApJ, 790, 133

Eastman, J., Gaudi, B. S., \& Agol, E. 2013, PASP, 125, 83

Elliott, P., Bayo, A., Melo, C. H. F., et al. 2014, A\&A, 568, A26

Feigelson, E. D., Lawson, W. A., Stark, M., Townsley, L., \& Garmire, G. P. 2006, AJ, 131, 1730

Foreman-Mackey, D., Hogg, D. W., Lang, D., \& Goodman, J. 2013, PASP, 125,306

Geweke, J. 1992, in Bayesian Statistics IV, ed. J. M. Bernardo (Oxford: Clarendon), 169

Goodman, J., \& Weare, J. 2010, Commun. Appl. Math. Comput. Sci., 5, 65

Henden, A. A., Levine, S. E., Terrell, D., Smith, T. C., \& Welch, D. 2012, JAVSO, 40, 430

Hillenbrand, L. A., \& White, R. J. 2004, ApJ, 604, 741

Holman, M., Touma, J., \& Tremaine, S. 1997, Natur, 386, 254
Horch, E. P., van Altena, W. F., Demarque, P., et al. 2015, AJ, 149, 151

Horch, E. P., Veillette, D. R., Baena Gallé, R., et al. 2009, AJ, 137, 5057

Janson, M., Bergfors, C., Brandner, W., et al. 2014, ApJS, 214, 17

Janson, M., Hormuth, F., Bergfors, C., et al. 2012, ApJ, 754, 44

Johnson, J. A., Clanton, C., Howard, A. W., et al. 2011, ApJS, 197, 26

Kasper, M., Apai, D., Janson, M., \& Brandner, W. 2007, A\&A, 472, 321

Kozai, Y. 1962, AJ, 67, 591

Kraus, A. L., Cody, A. M., Covey, K. R., et al. 2015, ApJ, 807, 3

Lidov, M. L. 1962, P\&SS, 9, 719

Liu, M. C., Wahhaj, Z., Biller, B. A., et al. 2010, Proc. SPIE, 7736, $77361 \mathrm{~K}$

Macintosh, B., Graham, J. R., Barman, T., et al. 2015, Sci, 350, 64

Malo, L., Doyon, R., Lafrenière, D., et al. 2013, ApJ, 762, 88

Mamajek, E. E., \& Bell, C. P. M. 2014, MNRAS, 445, 2169

Montet, B. T., Johnson, J. A., Muirhead, P. S., et al. 2015, ApJ, 800, 134

Moulds, V. E., Watson, C. A., Bonfils, X., Littlefair, S. P., \& Simpson, E. K. 2013, MNRAS, 430, 1709

Nielsen, E. L., \& Close, L. M. 2010, ApJ, 717, 878

Schaefer, G. H., Prato, L., Simon, M., \& Patience, J. 2014, AJ, 147, 157

Schlieder, J. E., Bonnefoy, M., Herbst, T. M., et al. 2014, ApJ, 783, 27

Shkolnik, E. L., Anglada-Escudé, G., Liu, M. C., et al. 2012, ApJ, 758, 56

Simon, M., \& Schaefer, G. H. 2011, ApJ, 743, 158

Stassun, K. G., Feiden, G. A., \& Torres, G. 2014, NewAR, 60, 1

Torres, C. A. O., Quast, G. R., Melo, C. H. F., \& Sterzik, M. F. 2008, in Handbook of Star Forming Regions, Volume II: The Southern Sky, ed.

B. Reipurth (San Francisco, CA: ASP), 757

van Leeuwen, F. 2007, A\&A, 474, 653

Wizinowich, P., Acton, D. S., Shelton, C., et al. 2000, PASP, 112, 315

Yelda, S., Lu, J. R., Ghez, A. M., et al. 2010, ApJ, 725, 331

Zuckerman, B., Song, I., \& Bessell, M. S. 2004, ApJL, 613, L65 